\title{
A utilização de dispositivos móveis para produção de Animações: Um relato de experiência
}

\author{
Janemar Hounsell Cruz ${ }^{1}$, Jonathas S. Sousa ${ }^{1}$, Thailane P. S. Neves ${ }^{1}$, Fernanda G.S. \\ Pires $^{1}$
}

\author{
${ }^{1}$ Escola Superior de Tecnologia - Universidade do Estado do Amazonas (UEA) \\ AV. Darcy Vargas 1200, Parque 10 de Novembro \\ CEP: 69050-20 - Manaus, AM - Brasil. \\ janemarhounsel@gmail.com, jonathassarmento50@gmail.com, \\ tpsn.influea.edu.br, fpires@uea.edu.br
}

\begin{abstract}
Resumo. $O$ artigo descreve um relato de experiência na produção de animações educativas nas aulas de Artes, usando tablets. Adotando a abordagem Construcionista/Construtivista, levando o aluno a desenvolver novas práticas de aprendizagem, por meio da produção de desenhos e animações frame-aframe, o que mostrou uma ótima aceitação tanto por parte dos alunos como do professor, que ficaram motivados a aderir essas tecnologias nas suas aulas.

Abstract: This paper shows an analysis of an experiment producing educational animations in the Arts classes, using tablets. Use as theoretical the constructionist / Constructivist approach, leading the student to develop new ways of learning, using the production of drawings and animations, frame-byframe. The work was well received and indicates that mobile devices can help in learning methods.
\end{abstract}

\section{Introdução}

A educação vem passando por mudanças em sua estrutura física e metodológica. Segundo Valente (1999), "A Educação é um serviço e, como tal, sofre e se adequa às concepções paradigmáticas que vive a sociedade" (p.32). E o que é mais questionado são os efeitos de tais mudanças, tanto na forma de pensar quanto de ensinar, por isso são traçados novos métodos de ensino/aprendizagem.

O mundo hoje produz inúmeras tecnologias, mas como levá-las para a sala de aula? É uma grande questão que professores enfrentam diariamente. Nesse cenário, encontramos o grande avanço no desenvolvimento de dispositivos móveis. Segundo a IDC Brasil, no começo do ano de 2014 foram vendidos 13 milhões de celulares inteligentes no Brasil (smartphone), mostrando assim um grande crescimento na utilização destes dispositivos.

Mobile Learning vem sendo defendida como uma alternativa viável de utilização de tecnologias móveis na educação como forma de construção de novos modelos pedagógicos visando à utilização de dispositivos móveis (celulares, tablets, PDA, etc) em processos educacionais (Ally \& Prieto-Blázquez, 2014; Cobcroft, Towers, Smith, \& Bruns, 2006; Traxler, 2005; Wu et al., 2012).

No que tange a realidade socioeducativa, a utilização de dispositivos móveis está presente no cotidiano de professores e alunos, permeando várias atividades do seu dia a dia. Para que esta ferramenta se torne um instrumento educacional, é necessário a qualificação dos educadores e o desenvolvimento de metodologias que utilizem esse 
contexto. O panorama educacional, a cada dia, ganha novos aliados tecnológicos que desencadeiam um processo de mudanças na estrutura metodológica que devem ser implementadas de acordo com as necessidades do contexto educacional.

O trabalho relatado envolve processos educativos e a utilização de dispositivos móveis na construção de projetos interdisciplinares, em uma escola pública, com 14 alunos do ensino fundamental I $5^{\mathrm{a}}$ Ano, com faixa etária entre 10 e 13 anos e dois professores das disciplinas de Artes e de Ciências. Tem-se como questão de pesquisa: "poderia a utilização de dispositivos móveis auxiliar o processo de produção de histórias animadas?".

Foram produzidas histórias animadas, sobre a temática "Problemas Ambientais" nas aulas de Artes, utilizando dispositivos móveis (tablets), o que configura uma abordagem interdisciplinar. Todo o processo produtivo foi realizado através de ferramentas para a plataforma Android de uso gratuito, sendo o Galaxy SketchBook usado para a produção de cenários e personagens e o FlipaClip para a produção de vídeos frame by frame.

O trabalho está organizado da seguinte forma: Discussão sobre o M-Learning, materiais e métodos em que se discutem as ferramentas, o relato em si, as discussões incluindo resultados e análises e as considerações finais.

\section{Trabalhos Relacionados}

Castral \& Vizioli (2011), relatam que ao utilizarem o Tablet no contexto escolar, alcançaram as metas no que tange ao aprimoramento de técnicas de desenho utilizando canetas de alta precisão (accurate pen).

Vieira \& Conforto, (2015) em seu relato de experiência, reafirmam a utilização de dispositivos móveis como ítens de identidade entre os jovens, bem como enfatizam a criação de material didático pedagógico utilizando estes dispositivos.

Medeiros e Pansanato (2015) em seus estudos, apontam a produção de vídeos que fujam do formato de aulas tradicionais como uma alternativa viável em otimização de processo de aprendizagem por valorizar a produção e a dinamicidade.

\section{M-Learning e o ensino multidisciplinar: Uma abordagem visual em Artes na escola}

Mobile Learning é definido pela MoleNET (2016) como "A exploração de tecnologias portáteis onipresentes (ubíqua), em conjunto com as redes de telefonia sem fio e móvel, para facilitar, apoiar, melhorar e ampliar o alcance do ensino e da aprendizagem ${ }^{1}$ ". Para Kukulska-Hume, (2005), a aprendizagem móvel trata da possibilidade de mobilidade dos alunos visando a possibilidade de envolvimento em atividades educacionais sem estar fisicamente vinculado a um local específico. Wu et al.,(2009) concluem que aprendizagem móvel diz respeito ao envolvimento de pessoas em atividades educacionais utilizando tecnologias como ferramentas de mediação da aprendizagem através de dispositivos móveis capazes de acessar dados e estabelecer comunicação com seus pares por meio de conexão sem fio.

\footnotetext{
${ }^{1}$ The exploitation of ubiquitous handheld technologies, together with wireless and mobile phone networks, to facilitate, support, enhance and extend the reach of teaching and learning.
} 
Aprendizagem com base em "Mobile Learning" ou, traduzindo, Aprendizagem móvel, tem como ideia principal a disponibilidade e alocação de conteúdos didático/pedagógicos por meio de dispositivos portáteis e de fácil acesso. Certal e Carvalho (2011) abrem uma discussão que é de todos: "poderá o M-Learning beneficiar o processo de aprendizagem no contexto educativo atual?".

Trabalhar com imagens tem um grande apelo cognitivo, por respeitar a forma do desenvolvimento das estruturas mentais (Beck \& Cunningham, 1989; de Souza, 2009; Van Merriënboer, Clark, \& De Croock, 2002; Van Merriënboer, Kirschner, \& Kester, 2003). Abordagem para aprendizagem de Ana Mae (2004) clarifica isso para o currículo

\begin{abstract}
A educação deveria prestar atenção ao discurso visual. Ensinar a gramática visual e sua sintaxe através da arte e tornar as crianças conscientes da produção humana de alta qualidade é uma forma de prepará-las para compreender e avaliar todo tipo de imagem, conscientizando-as de que estão aprendendo com estas imagens. Um currículo que interage atividades artísticas, história das artes e análise dos trabalhos artísticos levaria a satisfação das necessidades e interesses das crianças, respeitando ao mesmo tempo os conceitos da disciplina a ser aprendida, seus valores, suas estruturas e sua específica contribuição a cultura (Barbosa, 2004, p.47)
\end{abstract}

A apropriação de técnicas de produção de desenhos permite observar a leitura que os estudantes realizam do universo ao seu redor, sua produção artística evidencia o mundo a partir do seu olhar, trabalhar sob esse prisma com o conteúdo curricular de ciências possibilita uma abordagem curricular enriquecedora por consorciar técnicas de produção, conhecimento empírico e utilizar ferramentas tecnológicas que otimizam os processos intrínsecos.

\title{
3. Etapas de Pesquisa
}

O design experimental teve como público alvo alunos do $5^{\circ}$ ano, Ensino Fundamental I. Objetivo da ação: Criação de histórias animadas a partir dos seus constructos e experiências; Tema: problemas ambientais. A proposta usou como substrato teórico as Teorias Construtivistas de Jean Piaget (1977) e a Construcionista de Seymour Paper (2008), que aposta no protagonismo do estudante como construtor do seu conhecimento a partir das reflexões e desafios que lhe são propostos.

Os estudantes produziram material didático digital a partir do desenvolvimento de competências e habilidades partindo de seus constructos pessoais, ação defendida como benéfica (Malan \& Leitner, 2007). As atividades tiveram início através de dados colhidos pela observação e avaliação diagnóstica realizada através de duas etapas: aplicação de formulário estruturado a fim de reconhecer a condição socioeconômica dos alunos, sua exposição a tecnologias digitais, entre outros itens; a segunda etapa deu-se por meio de consulta aberta, pesquisa participativa, visando selecionar a melhor forma para desenvolver as atividades usando as ferramentas que até o momento eram novas para os alunos.

\subsection{Ferramentas utilizadas:}

$\checkmark$ Computador pessoal (monitor, gabinete e mouse) com sistema operacional Windows, Software de desenho nativo do mesmo.

$\checkmark$ Tablet com caneta para escrita;

$\checkmark$ FlipaClip: Aplicativo gratuito, plataforma Android, desenvolvido no âmbito de um concurso de aplicativos com caneta e escrita para tablete. Permite a criação de 
desenhos animados com elementos criados por qualquer pessoa. Os desenhos quadro a quadro remontam a ideia de cadernos de desenho, o app mostra ainda a sombra do desenho anterior, possibilitando ao usuário seguir traços realizando pequenas mudanças a fim de dar mobilidade às gravuras.

$\checkmark$ SketchBook Spen- Aplicativo gratuito Autodesk desenvolvido especialmente para a linha Note da Samsung. Possibilita a criação de desenhos em camadas, bem como possui uma coleção de pincéis e ferramentas que facilitam a tarefa de composição.

\subsection{A experiência de utilização de TIC aplicadas à educação}

Um dos primeiros dados de observação colhida foi à prática de sala de aula no conteúdo curricular de Ciências. O professor utiliza o computador para que os estudantes criem expressões do material trabalhado nas aulas expositivas. Em uma aula, os mesmos utilizaram uma ferramenta de desenho nativa do sistema operacional Windows para criar ilustrações sobre o tema "os primeiros seres vivos da Terra". A tarefa durou em média 40 minutos, os alunos demonstraram grande dificuldade em trabalhar com o mouse e seus traços denotam falta de acurácia no desenvolvimento da tarefa. Mesmo depois desse tempo, a atividade não pode ser concluída no tempo de aula proposto. Quando questionados sobre a dificuldade encontrada em trabalhar com o mouse, $88 \%$ informaram ter grandes dificuldades em trabalhar com o mesmo.

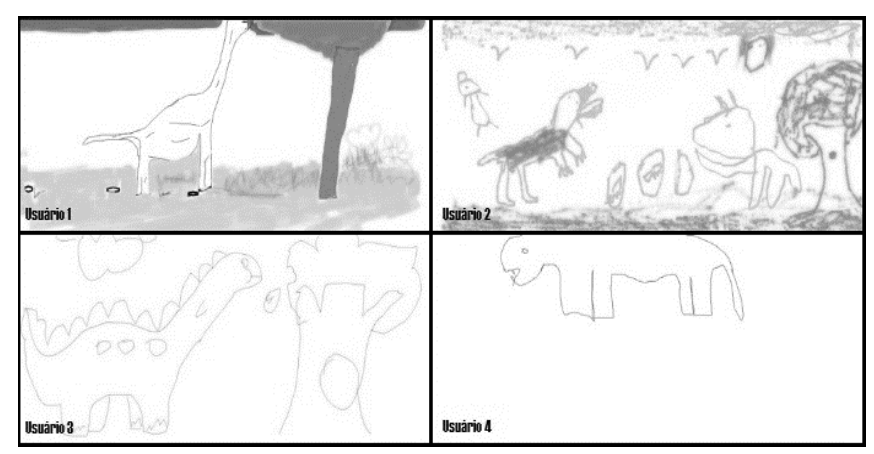

Figura 1: Atividade primeiros seres vivos 5a Ano

O alto nível de engajamento utilizado na ferramenta tende a reduzir a carga cognitiva que poderia ser utilizada para a retenção do objeto de estudo (Mayer, 2009). A Figura 1 mostra que os alunos demostraram dificuldade nas ações que envolvem a coordenação motora fina. Segundo Pellegrini, habilidades motoras finas são:

[...] aquelas que requerem muita precisão, envolvem principalmente os
membros superiores, em específico as mãos. Um grande número de músculos,
relativamente pequenos, é ativado na execução destas habilidades.
Encontramos no rol de habilidades motoras finas, o escrever, o digitar, o fazer
crochê, ou consertar um relógio e, mais precisamente no processo de
alfabetização, o discernimento entre escrever as letras que são parecidas
graficamente: $\mathrm{m} / \mathrm{n}, \mathrm{g} / \mathrm{q}, \mathrm{l} / \mathrm{b}$, dentre outras. (Pellegrini ,2003, p.181)

Por meio de testes de escrita e elaboração de desenhos, junto aos alunos, observamos à escrita e a coordenação motora (desenhos). Muitos estudantes na sala de aula têm dificuldades em relação aos dois casos. O professor da disciplina disse, em entrevista, que os alunos apresentavam dificuldades em relação à coordenação motora (desenhos) e a escrita. A pesquisa informa que 64\% da turma apresentaram dificuldades na escrita e $70 \%$ mostrou problemas com relação à coordenação motora. 
A segunda forma de coleta de dados deu-se através da aplicação de questionário. Ação realizada por meio de formulários digitais. Os dados colhidos demonstram que $94 \%$ dos alunos possuem televisão em casa, $64 \%$ possuem rádios, $41 \%$ possuem TV por assinatura, $41 \%$ possuem computador em casa, $52 \%$ possuem acesso à internet e $100 \%$ dos alunos questionados possuem Smartphones em casa. Outro quesito analisado foi a frequência com que esses alunos têm acesso a jogos eletrônicos, 52\% informaram que tem acesso durante 7 dias por semana e $47 \%$ usam pelo menos 3 vezes a cada 7 dias. Quanto à frequência de acesso ao smartphone, 66\% informaram que tem acesso durante todos os dias da semana.

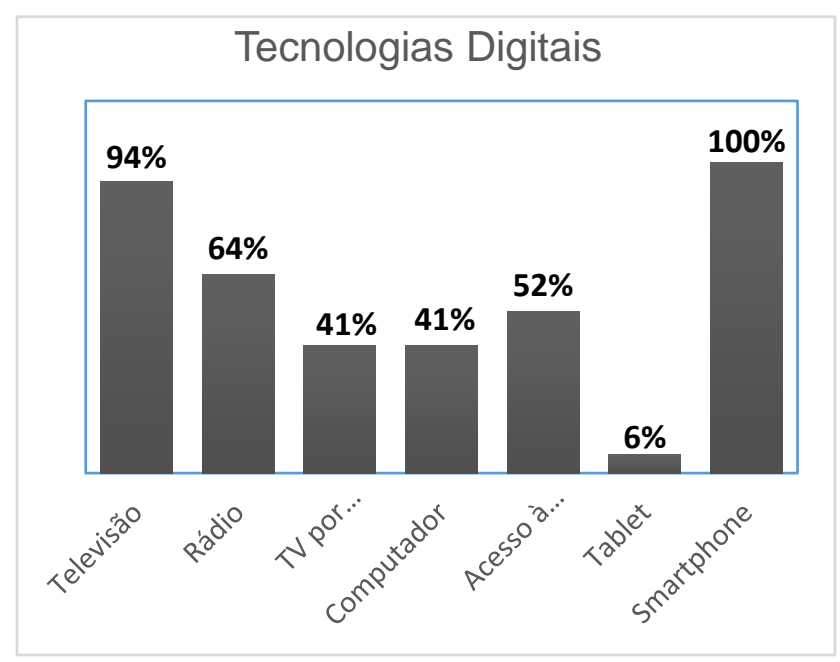

\section{Gráfico 1: Tecnologias presentes na residência dos alunos}

Visualmente, tinham acesso ininterrupto a dispositivos móveis digitais e 100\% conhecia e tinha smartphones em casa. Informação que serve de aporte a proposta de trabalho com dispositivos móveis.

Quanto a propensão, 94,1\% dos entrevistados afirmaram gostar de desenhar e somente 5,9\% não gostavam da atividade, quando questionados sobre a possibilidade de realizar desenhos "manuais" em tablets, em aulas futuras, todos se mostraram abertos e desejosos de realizar tal atividade.

\subsection{Configuração e uso do Ambiente de testes}

A aplicação foi realizada no laboratório de dispositivos móveis composto por: um servidor, dois roteadores, um rack contendo 40 tablets com processadores octa-core, câmera integrada e canetas para escrita em tela. O primeiro passo foi a apresentação de um tutorial sobre o aplicativo SketchBook, para isso os alunos desenvolveram atividades usando basicamente dois recursos digitais, o lápis e a borracha. Depois do contato inicial lhes foi solicitado que criassem um cenário que contivesse uma morada.

O uso da tecnologia em sala de aula foi bem aceito pelos alunos que demoraram em média 50 minutos para realizar a atividade, pois ainda tinham tido pouca experiência com o dispositivo móvel. Dados observacionais apontam que todos os alunos tentaram criar seus desenhos como se estivessem desenhando em uma folha, evitando figuras geométricas e objetos pré-existentes no aplicativo.

No segundo dia, foi pedido aos alunos que realizassem a mesma tarefa do dia anterior, mas dessa vez deveriam utilizar camadas, preenchimentos, pincéis artísticos e 
V Congresso Brasileiro de Informática na Educação (CBIE 2016)

Anais dos Workshops do V Congresso Brasileiro de Informática na Educação (CBIE 2016)

figuras geométricas pré-existentes na ferramenta. Apesar da complexidade, com relação à ferramenta ser maior que no dia anterior, a tarefa foi cumprida em 30 minutos, pouco mais que a metade do tempo do dia anterior, e menor do que o gasto no desktop. Na produção, fica clara a evolução, como demonstrado nas ilustrações abaixo.

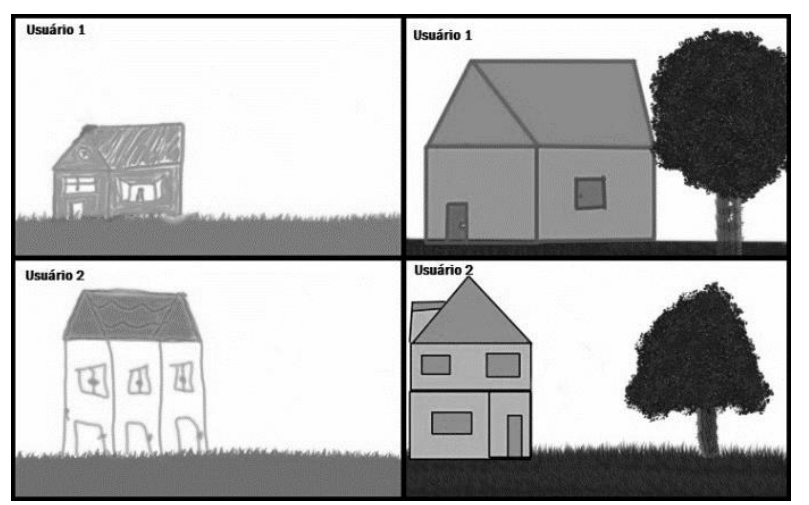

Figura 3: Comparativos entre $1^{\circ}$ e $2^{\circ}$ dia

$\mathrm{O}$ terceiro e o quarto momento (dia) foram dedicados ao produto final. Primeiro lhes foi solicitado que escolhessem o tipo de poluição ambiental que gostariam de desenhar. SketchBook serviu para que criassem cenários para futura animação.

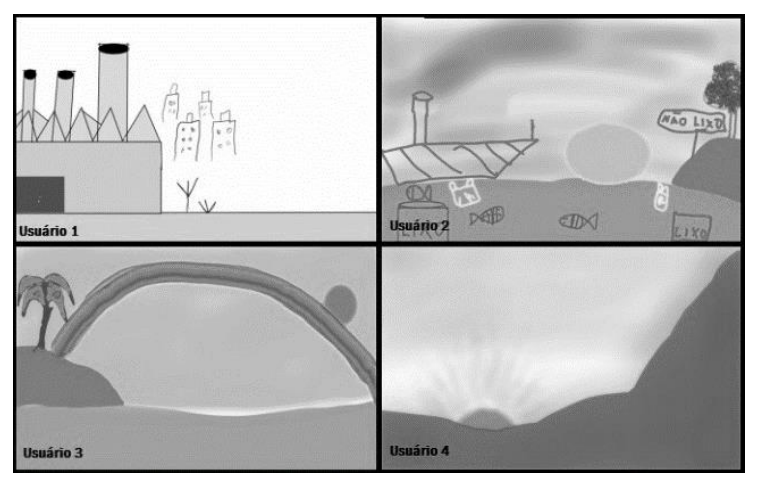

Figura 4: Cenários finais para o projeto

Para finalizar os trabalhos, após a elaboração dos cenários baseados no tema poluição ambiental feito no aplicativo SketchBook, os alunos passaram a estudar conceitos básicos de animações e como usar o FlipaClip com todas suas funcionalidades. Os cenários foram exportados para que pudessem fazer suas animações frame-a-frame usando os tablets com caneta facilitando o desenho de todos os quadros. 


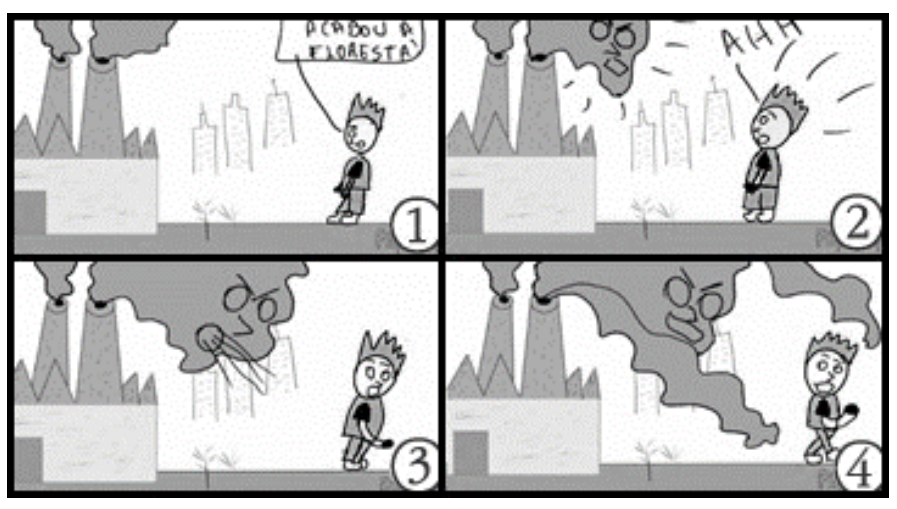

Figura 5: Projeto no FlipaClip frame-a-frame

\section{Resultados e discussões}

Durante a pesquisa constatou-se que os dispositivos móveis podem auxiliar no processo de ensino aprendizagem. Os alunos envolvidos apresentaram um índice de satisfação de $85,77 \%$ e $100 \%$ dos professores questionados acreditam que os dispositivos móveis podem auxiliar nos processos educacionais.

Os resultados do trabalho apontam que os tablets são alternativas viáveis para a criação de material didático pedagógico próprio pois a apropriação da ferramenta (hardware/software) apresentou uma curva de aprendizagem relativamente menor do que encontrada em trabalhos semelhantes realizados em desktop.

Observamos que a mobilidade da ferramenta e a inexistência de dispositivos com que os alunos não tem muito contato, como o computador de mesa com seus periféricos, fez com que os estudantes tivessem mais "intimidade" com os tablets, usando-os como cadernos, não apresentando a mesma dificuldade encontrada no uso dos mouses. Como defende Kukulska-Hume (2005) quando um dispositivo tem boa usabilidade, a aprendizagem segue sem obstáculos e pode ser otimizada pela existência de determinados recursos.

Foi comparado o tempo gasto com as atividades desenvolvidas primeiramente no computador depois com o tablet, fazendo desenhos livres e com formas pré-definidas. As atividades com os tablets tem um grande ganho de tempo e aquisição de competências, pois consideramos que todas as atividades foram realizadas no tempo proposto. Consideramos uma ganho também o produto final, na forma de animação.

O uso do tablet funde-se com a experiência que os estudantes já têm nos smartphones de suas casas, como vimos, $100 \%$ dos alunos possuía acesso mesmo que indireto a ferramenta, e $41 \%$ dos entrevistados possuía computadores em casa. A utilização de canetas de grande acurácia auxilia no processo de melhoria da coordenação motora. Quando o aluno trabalha com o tablet, o mesmo está transpassando o que realiza quando desenha em seu caderno, para ele a dificuldade em trabalhar a coordenação motora diminui, por contar com uma gama de ferramentas que facilitam todo o processo.

\footnotetext{
"A coordenação motora é definida como junção de um conjunto de habilidades e das estruturas corporais. Dentro dos pré-requisitos para o desenvolvimento da coordenação motora, encontram-se a experiência adquirida, a informação sensorial, a capacidade intelectual e a antecipação. Em algumas habilidades, a coordenação se manifesta muito antes do que em outras". (Rodriguez, 2005, p.191)
} 
Para realizar atividades de construção de animações, normalmente seriam necessários softwares sofisticados, bem como uma mesa digitalizadora que permitisse criar os desenhos além de um computador capaz de realizar processamento de vídeo. No experimento aqui demonstrado, um único dispositivo conseguiu reunir todos os elementos, possibilitando atingir os objetivos educacionais em sala de aula.

\section{Considerações Finais}

As análises relizadas apontam que a utilização de dispositivos móveis associados a tarefas educacionais em ambiente escolar podem otimizar processos, aumentar o engajamento dos alunos, aumentar o tempo passado entre estudantes e seu objeto de estudo criando possibilidade de gerar uma aprendizagem significativa. A produção de animações permitiu aos estudantes criarem os seus próprios objetos de aprendizagem o que mostrouse enriquecedor do ponto de vista pedagógico. O nível de aceitação demonstrado por professores e alunos nos permite indicar a utilização de métodos que tenham espaço para a utilização de Mobile Learning. A partir dos dados analisados durante o trabalho fica clara a importância do uso da ferramenta na execução de desenhos, cenários e animações. O tempo gasto e o conforto dado pelos dispositivos para desenhar fez com que os alunos demostrassem um bom índice de satisfação com trabalho.

Trabalhar sob uma perspectiva multidisciplinar utilizando a produção artística e tecnologia mostrou ser produtivo, e para além do currículo de Ciências, por permitir uma abordagem protagonizada pelos estudantes, abrindo espaço para sua visão e leitura do mundo. O componente curricular de Artes abre espaço para a criatividade, o produto gerado pelos estudantes, a partir de análises observacionais, possibilita a identificação de quadros sociais específicos, enriquecendo o espaço educacional.

O uso de dispositivos e aplicativos não se restringe somente as disciplinas de artes e ciências, cabendo aos educadores buscar novas formas de trabalhar essas tecnologias. Uma mudança metodológica pode ocorrer, assim como uma reflexão coletiva na escola, para que as alterações sejam postas em prática.

Em suma, o objetivo de melhorar o ensino, usando ferramentas tecnológicas, registrou um resultado positivo, bem como a questão de pesquisa inicialmente levantada sendo esta comprovada.

\section{Referências Bibliográficas}

Ally, M. (2009). Mobile learning: Transforming the delivery of education and training. Athabasca University Press.

Ally, M., \& Prieto-Blázquez, J. (2014). What is the future of mobile learning in education? Revista de Universidad y Sociedad del Conocimiento, 11(1), 142-151.

Barcelos, R., Tarouco, L., \& Bercht, M. (2009). O uso de mobile learning no ensino de algoritmos. RENOTE, 7(3), 327-337.

Barbosa, A. M. (2004). Arte, educação e cultura. Portal Domínio Público. Recuperado de http://www. dominiopublico. gov. br/pesquisa/DetalheObraDownload. 
V Congresso Brasileiro de Informática na Educação (CBIE 2016)

Anais dos Workshops do V Congresso Brasileiro de Informática na Educação (CBIE 2016)

Beck, K., \& Cunningham, W. (1989). A laboratory for teaching object oriented thinking. Paper presented at the ACM Sigplan Notices.

Castral, P. C., \& Vizioli, S. H. T. (2011). O desenho à mão livre mediado pela tablet. Anais do Sigradi: cultura aumentada.

Certal, F. M., \& Carvalho, A. A. A. (2011). Estudo sobre receptividade ao m-learning no ensino básico. In VII Conferência Internacional de TIC na Educação-Challenges 2011-Perspectivas de Inovação (pp. 1427-1438).

Idc Brasil.(2014). International Data Corporation. Estudo da IDC aponta recorde de vendas de smartphones no Brasil no segundo trimestre de 2014. São Paulo: IDC Brasil, Recuperado de http://br.idclatin.com/releases/news.aspx?id=1713

Lima Medeiros, S. F., \& Pansanato, L. (2015). Estudo das Preferências de Alunos e Professores sobre Vídeo aula para Identificar Requisitos de Software para Ferramentas de Produção. In Anais do Simpósio Brasileiro de Informática na Educação (Vol. 26, No. 1, p. 219).

Diretoria de Pesquisas. (2011). Coordenação de Trabalho e Rendimento. Pesquisa Nacional por Amostra de Domicílios. Recuperado de http://www.ibge.gov.br/h ome/estatistica/po pulacão /acessoainternet2011/default.shtm.

Junior, J. B. B., Coutinho, C., \& Alexandre, D. S. (2006). M-Learning e Webquests: as novas tecnologias como recurso pedagógico. In: Anais do Simpósio Brasileiro de Informática na Educação (Vol. 1, No. 1, pp. 70-72).

Kukulska-Hulme, A. (2005). Mobile usability and user experience. Mobile Learning: $A$ handbook for educators and trainers, 45-56.

Malan, D. J., \& Leitner, H. H. (2007). Scratch for budding computer scientists. Paper presented at the ACM SIGCSE Bulletin.

Maloney, J. H., Peppler, K., Kafai, Y., Resnick, M., \& Rusk, N. (2008). Programming by choice: urban youth learning programming with scratch (Vol. 40): ACM.

Mayer, R. E. (2009). Teoria Cognitiva da Aprendizagem Multimédia. In G. L. Miranda (Ed.), Ensino online e aprendizagem multimédia (pp. 207-237). Lisboa: Relógio D'Água.

Meirelles, L. F. T., Tarouco, L. M. R., \& da Silva, G. D. G. (2006). Aprendizagem com mobilidade para as atividades de prática em cursos de Licenciatura. RENOTE, 4(1).

Pellegrini, A. M., Neto, S. S., Bueno, F. C. R., Alleoni, B. N., \& Motta, A. I. (2005). Desenvolvendo a coordenação motora no ensino fundamental. São Paulo: UNESP.

Pelissoli, L., \& Loyolla, W. (2004). Aprendizado móvel (M-Learning): dispositivos e cenários. In Actas do congresso Internacional de Educação a Distância, Brasil. Recuperado de http://www.abed.org.br/congresso2004/por/htm/074-TC-C2. htm Acessivel em (Vol. 15, pp. 03-06). 
V Congresso Brasileiro de Informática na Educação (CBIE 2016)

Anais dos Workshops do V Congresso Brasileiro de Informática na Educação (CBIE 2016)

Papert, S.(2008). A máquina das crianças: repensando a escola na era da informática. Porto Alegre : Artmed, 2008.

Piaget, J., \& Figueiredo, Á. (1977). O desenvolvimento do pensamento: equilibração das estruturas cognitivas. Lisboa: Dom Quixote

Resnick, M., Maloney, J., Monroy-Hernández, A., Rusk, N., Eastmond, E., Brennan, K.,... Silverman, B. (2009). Scratch: programming for all. Communications of the ACM, 52(11), 60-67.

Rodríguez, C. G. (2005). Educação física infantil: motricidade de 1 a 6 anos. São Paulo: Editora Phorte

Souza, C. M. (2009). VisuAlg-Ferramenta de apoio ao ensino de programação. Revista Eletrônica TECCEN, 2(2), 01-09.

Shneiderman, B. (1993). 1.1 direct manipulation: a step beyond programming languages. Sparks of innovation in human-computer interaction, 17, 1993.

Traxler, J. (2005). Defining mobile learning. In IADIS International Conference Mobile Learning (pp. 261-266).

Valente, J. A. (1999). O computador na sociedade do conhecimento. Mudanças na sociedade, mudanças na Educação: o fazer e o compreender. Campinas: Editora Unicamp/NIED.

Van Merriënboer, J. J., Clark, R. E., \& De Croock, M. B. (2002). Blueprints for complex learning: The 4C/ID-model. Educational technology research and development, 50(2), $39-61$.

Van Merriënboer, J. J., Kirschner, P. A., \& Kester, L. (2003). Taking the load off a learner's mind: Instructional design for complex learning. Educational psychologist, 38(1), 513.

Vieira, M., \& Conforto, D. (2015). Aprendizagem Móvel e Multimídia: a produção de material pedagógico na perspectiva BYOD. Paper presented at the Anais do Workshop de Informática na Escola.

Wu, W. H., Wu, Y. C. J., Chen, C. Y., Kao, H. Y., Lin, C. H., \& Huang, S. H. (2012). Review of trends from mobile learning studies: A meta-analysis.Computers \& Education, 59(2), 817-827. 